\title{
Prevalence and Determinants of Intestinal Parasitic Infections among Pregnant Women Receiving Antenatal Care in Kasoa Polyclinic, Ghana
}

\author{
Albert Abaka-Yawson $\left(\mathbb{D},{ }^{1,2}\right.$ Solomon Quarshie Sosu, ${ }^{2}$ Precious Kwablah Kwadzokpui, \\ Salomey Afari, ${ }^{3}$ Samuel Adusei, ${ }^{4}$ and John Arko-Mensah ${ }^{1}$ \\ ${ }^{1}$ Department of Biological, Environmental \& Occupational Health Sciences, School of Public Health, University of Ghana, \\ Legon, Ghana \\ ${ }^{2}$ Department of Medical Laboratory Sciences, School of Allied Health Sciences, University of Health and Allied Sciences, \\ Ho, Ghana \\ ${ }^{3}$ Department of Pharmaceutics, School of Pharmacy, University of Health and Allied Sciences, Ho, Ghana \\ ${ }^{4}$ Department of Obstetrics and Gynaecology, University of Cape Town, Cape Town, South Africa \\ Correspondence should be addressed to Albert Abaka-Yawson; aabakayawson@uhas.edu.gh
}

Received 31 March 2020; Revised 15 August 2020; Accepted 28 August 2020; Published 8 September 2020

Academic Editor: Jonathan Haughton

Copyright ( 2020 Albert Abaka-Yawson et al. This is an open access article distributed under the Creative Commons Attribution License, which permits unrestricted use, distribution, and reproduction in any medium, provided the original work is properly cited.

\begin{abstract}
Background. Intestinal parasitic infections affect pregnant women worldwide. The infection has been implicated in causing lifethreatening conditions in both gravid women and their developing foetus. Sub-Saharan Africa is known to harbor the greatest proportion of intestinal parasitic infections largely due to socioeconomic and environmental factors. In Kasoa, Southern Ghana, there is paucity of data on the prevalence and associated factors of intestinal parasitic infections among pregnant women. Objective. The aim of the study was to determine the prevalence of intestinal parasitic infections and associated factors among pregnant women attending antenatal care in Kasoa Polyclinic. Methods. A hospital based analytical cross-sectional study was carried out among three hundred (300) conveniently sampled pregnant women receiving antenatal care services at the Kasoa Polyclinic. Structured questionnaires were administered to the study participants to assess sociodemographic and other possible factors. Stool samples were collected from each pregnant woman and examined for the presence of intestinal parasites by microscopy using direct wet mount as well as formol-ether sedimentation techniques. Results. Overall prevalence of intestinal parasites was 14.3\% (95\% CI 11-19\%). Entamoeba histolytica (5.0\%) was the most predominant parasite species identified followed by Ascaris lumbricoides (4.3\%), Giardia lamblia (2.3\%), Trichuris trichiura (1.3\%), Schistosoma mansoni (0.3\%), Hookworm (0.3\%), Hymenolepis nana (0.3\%), and Isospora belli $(0.3 \%)$. Age $>30$ years $(\mathrm{AOR}=0.17,95 \% \mathrm{CI}=0.06-0.48$; $p=0.001)$, multigravidity $(\mathrm{AOR}=0.43,95 \% \mathrm{CI}=0.19-0.97 ; p=0.043)$, and $2^{\text {nd }}$ and $3^{\text {rd }}$ trimesters $(\mathrm{AOR}=4.73,95 \%$ $\mathrm{CI}=1.36-16.49 ; p=0.015)$ were independently associated with intestinal parasitic infections among pregnant women. Conclusions. A prevalence of $14.3 \%$ pregnant women compared to previous studies in Ghana is relatively low. It however suggests that intestinal parasitic infection is still a problem. The major factors noted were age, gravidity, and gestational age. Routine stool examination and provision of public health education are recommended to prevent infection of pregnant mothers and their unborn babies.
\end{abstract}

\section{Introduction}

Intestinal parasitic infections are primarily caused by protozoans and helminthes [1]. They are frequently transmitted via consumption of contaminated food, bathing, and wading through contaminated water as well as spread from person to person through fecal-oral contact. Intestinal parasitic infections are associated with socioeconomic and environmental 
factors. They are therefore prevalent in areas where there is overcrowding, limited access to clean water, and poor personal hygiene [2, 3].

Pregnant women, especially those in Africa, are at greater risk of intestinal parasitic infections [4]. A recent study in Ethiopia found $70.6 \%$ (553/783) pregnant women to be infected with intestinal parasites with helminthes being the predominant species [5]. In Nigeria, it was observed that $18.2 \%$ (73/401) pregnant women were living with intestinal parasitic infections [6]. Data from previous studies in Ghana showed that intestinal parasitic infections among pregnant women were $41.2 \%$ [7] and $49.6 \%$ [8]. Variations in geographical location and hygienic practices have been identified as important contributing factors to prevalence of intestinal parasitic infections in any population.

Infection with intestinal parasites such as hookworms is known to cause anemia in pregnant women [9], contributing to adverse pregnancy outcomes such as low birth weight and impaired milk production. The roundworm, Ascaris lumbricoides, infection has also been implicated in diminished food intake and weight loss in pregnant women [3]. Furthermore it has also been reported to affect survival, growth, and cognitive performance of children born to infected mothers [10].

Additionally, the propensity of trophozoites of the protozoan parasite, Entamoeba histolytica to invade the intestinal mucosa, the liver, lungs, and blood circulation of the human host leads to abdominal discomfort, pulmonary abscesses, bleeding episodes, and sometimes death if not diagnosed early for timely treatment [11].

This presents a significant public health situation that requires urgent attention. To deal with this appropriately, the Ministry of Health in collaboration with the Ghana Health Service set up the Neglected Tropical Diseases Control Program (NTDCP) with the rationale of alleviating the occurrence of neglected tropical diseases including intestinal parasitic infections in the country to an insignificant level [12]. This study aimed to determine the prevalence and determinants of intestinal parasitic infections among pregnant women attending antenatal care in Kasoa Polyclinic.

\section{Materials and Methods}

2.1. StudyDesign/Site. A hospital based cross-sectional study using convenient sampling was carried out on pregnant women attending routine antenatal care and laboratory department of the Kasoa Polyclinic. Kasoa is the capital of the Awutu Senya East municipality in the Central Region of Ghana. It has a total of about 69,384 people, which represents more than 79 times its population 40 years ago [13]. Kasoa has main regional market and is known to be a city that for some time has been battling with issues of overcrowding, limited access to treated water, and sanitation facilities [14].

2.2. Data Collection and Eligibility Criteria. Closed ended structured questionnaires were then administered with the aid of a local language translator to collect data on their sociodemographic characteristics and potential contributing factors for intestinal parasitic infections. Additionally, pregnant women at various stages of pregnancy were recruited at the Kasoa Polyclinic during attendance to antenatal care services. Pregnant women who have been on any form of antihelminthic therapy within the past three weeks were exempted from the study. Clean screw capped plastic stool containers with wide neck were given to the study participants. The stool containers were identified by prelabeling them with unique identification numbers. The study participants were requested to provide about $2 \mathrm{~g}$ (a small spatula attached to the stool container) of stool sample within 24 hours and examined using the direct wet mount method as well as the formol-ether concentration technique for the presence of parasites (trophozoites, cysts, ova, and larvae).

2.3. Sample Size. The prevalence of parasitic infections among pregnant women was determined from a Ghanaian study by Fuseini et al. [15] to be $23.0 \%$. The prevalence rate from that study was used to calculate the sample size to be 272. A final sample size of 300 study participants was chosen to account for nonresponse illustrated as follows.

The sample size for the study was calculated using Fisher's sampling formula:

$$
N=\frac{Z^{2} P(1-P)}{D^{2}}
$$

where $N$ represents the estimated sample size, $Z$ represents the constant for $95 \%$ confidence interval given as 1.96, $P$ represents the average prevalence of intestinal parasitic infections of $23.0 \%$ obtained from a study conducted among pregnant women in Kassena-Nankana district of the Northern Region, and $D$ represents the percentage margin of error taken as $5 \%$

\section{Analysis of Stool Samples}

3.1. Direct Wet Mount Method. About half of the $2 \mathrm{~g}$ stool sample was processed using the direct wet mount method by emulsifying the stool in a normal saline. A drop of the emulsified stool sample was then placed on a labelled glass slide and covered with a coverslip. The preparation was first examined under a 10x objective lens and then $40 \mathrm{x}$ for identification of parasites under low light intensity.

3.2. Formol-Ether Concentration Technique. For formolether concentration technique, about one gram $(1 \mathrm{~g})$ of each stool sample was well emulsified with $4 \mathrm{ml}$ of $10 \%$ formol saline into a $15 \mathrm{ml}$ conical centrifuge tube through wet cheesecloth-type gauze placed over a disposable paper funnel. The mixture was centrifuged at $3000 \mathrm{rpm}$ for five (5) minutes and the supernatant decanted after centrifugation. An additional $4 \mathrm{ml}$ of $10 \%$ formol saline and a next four (4) $\mathrm{ml}$ of diethyl ether were added to the sediment, mixed adequately, and then subjected to a second round of centrifugation at $3000 \mathrm{rpm}$ for 5 minutes. The supernatant was then discarded and the concentrated sediments resuspended in $10 \%$ formol saline. The 
sediments were examined microscopically under 10x and 40x magnification for the presence of parasitic organisms as described by Cheesbrough [16].

3.3. Quality Control. Known positive and negative slides for the various intestinal parasites were tested using the direct wet mount method and formol-ether concentration method as indicated in the laboratory procedure. Samples and slides were declared positive when various stages of the parasites, such as trophozoites, cysts, ova, and larvae, were observed. This was to ensure reliability of the test procedure as well as a refresher course for the researcher. Additionally, two expert parasitologists were employed to assist with the microscopy.

3.4. Ethical Considerations. Protocols used for the study were approved by the Ghana Health Services Ethics Review Committee (Protocol number: GHS-ERC: 067/12/17) and permission was also granted by the Kasoa Polyclinic to carry out the study. Informed written consent was sought from the study participants with the objectives and procedures clearly stated in the consent form. Further, the data collected was treated with enough confidentiality during collection, during analysis, and even after analysis. Possibility of withdrawal from the study at any point was made known to the study participant. Additionally, pregnant women who tested positive were referred to physicians for appropriate medical attention.

3.5. Data Analysis. Data collected from the questionnaires and results from the laboratory analysis was checked for consistency and correctness and entered into Microsoft Excel software using fit-for-purpose excel form so as to avoid as much as possible entry errors. The compiled data was analyzed using Stata SE version 15 (StataCorp., College Station, TX, USA). Data is presented as frequencies and standard deviations. The magnitude of association between intestinal parasitic infections and potential risk factors was assessed using univariate and multivariate logistic regression and described in terms of crude odds ratio (cOR) and adjusted odds ratio (aOR) at $95 \%$ confidence interval. $p$ values of $<0.05$ were considered statistically significant.

\section{Results}

4.1. General Characteristics of Study Participants. The study recruited three hundred (300) pregnant women majority (62.0\%) of which were aged 21-30 years and the next most predominant category $(32.0 \%)$ falling within the age bracket of 31-40 years. Fifty-seven (19.0\%) of the women had no formal education, whereas $25.0 \%$ (75/300) were able to make it up to their primary education level. In the preponderance of the pregnant women, 116 (38.7\%) had their junior high school education with smaller proportions attaining their senior high school tertiary education. More than half $(76.7 \%)$ of the study participants were married, while $68.3 \%$ were gainfully employed.
Predominantly, $57.3 \%$ and $73.7 \%$ of the pregnant women were primigravidae and in their late trimesters of pregnancy, respectively, possessed their own toilet facility $78 \%$ (234/ $300)$, and practiced hand washing (96.3\%) before meals. A good number of the participants also indicated that they prepared their food at home (85.7\%) and drank water from treated sources $(96.3 \%)$. The study recorded majority, 212 (70.7\%), of the participants who indicated that they had dewormed for over 6 months at the time of the study (Table 1).

4.2. Prevalence of Intestinal Parasitic Infections. Out of the 300 pregnant women whose stool samples were examined by direct wet mount and formol-ether concentration techniques, $43(14.3 \%)$ of them had intestinal parasites. Out of this, $23(7.7 \%)$ participants had intestinal protozoan parasites and $20(6.7 \%)$ were infected by intestinal helminthes.

The predominant protozoan parasite was Entamoeba histolytica which accounted for 15 (5.0\%) of infections among pregnant women. This was followed by Giardia lamblia, 7 (2.3\%), and Isospora belli, 1 (0.3\%).

With regard to helminthic infections, the predominant parasite species was Ascaris lumbricoides with a prevalence of 13 (4.3\%), followed by Trichuris trichiura 4 (1.3\%). Other parasites seen were Schistosoma mansoni $1(0.3 \%)$, Hymenolepis nana $1(0.3 \%)$, and Hookworm 1 (0.3\%) (Figure 1).

4.3. Sociodemographic Factors Associated with Intestinal Parasitic Infections. Table 2 shows the logistic regression analysis of sociodemographic characteristics stratified by intestinal parasitic infections. Three factors were identified to be associated significantly with intestinal parasitic infections by the univariate logistic regression analysis. These include age $(\mathrm{cOR}=0.22 ; 95 \% \mathrm{CI}=0.83-1.57 ; p=0.002)$, education ( $\mathrm{cOR}=0.41 ; 95 \% \mathrm{CI}=0.21-0.80 ; p=0.009)$, and employment status $\quad(\mathrm{cOR}=0.38 ; \quad 95 \% \quad \mathrm{CI}=0.20-0.73$; $p=0.004)$.

After adjusting for potential confounders using multivariate logistic regression analysis, only age showed a statistically significant association with intestinal parasitic infections. Pregnant women above 30 years had $83 \%$ reduced odds of having intestinal parasitic infections compared to those who were 30 years and below $(\mathrm{AOR}=0.17$; $95 \% \mathrm{CI}=0.06-0.48 ; p<0.001)$.

4.4. Pregnancy-Related and Behavioral Factors Associated with Intestinal Parasitic Infections. In the univariate analysis, factors such as type of toilet facility used, hand washing before meals, and drinking water sources were significantly associated with intestinal parasitic infections among pregnant women. Pregnant women who shared a toilet facility had 2.78-fold greater odds of intestinal parasite infections compared to those who owned a toilet facility $(\mathrm{COR}=2.78$; 95\% CI $=1.40-5.51 ; p=0.004$ ).

Pregnant women who washed their hands before meals had $82 \%$ reduced odds of intestinal parasite infections compared to their counterparts who did not $(\mathrm{COR}=0.18 ; 95 \%$ 
TABLE 1: General characteristics of study participants.

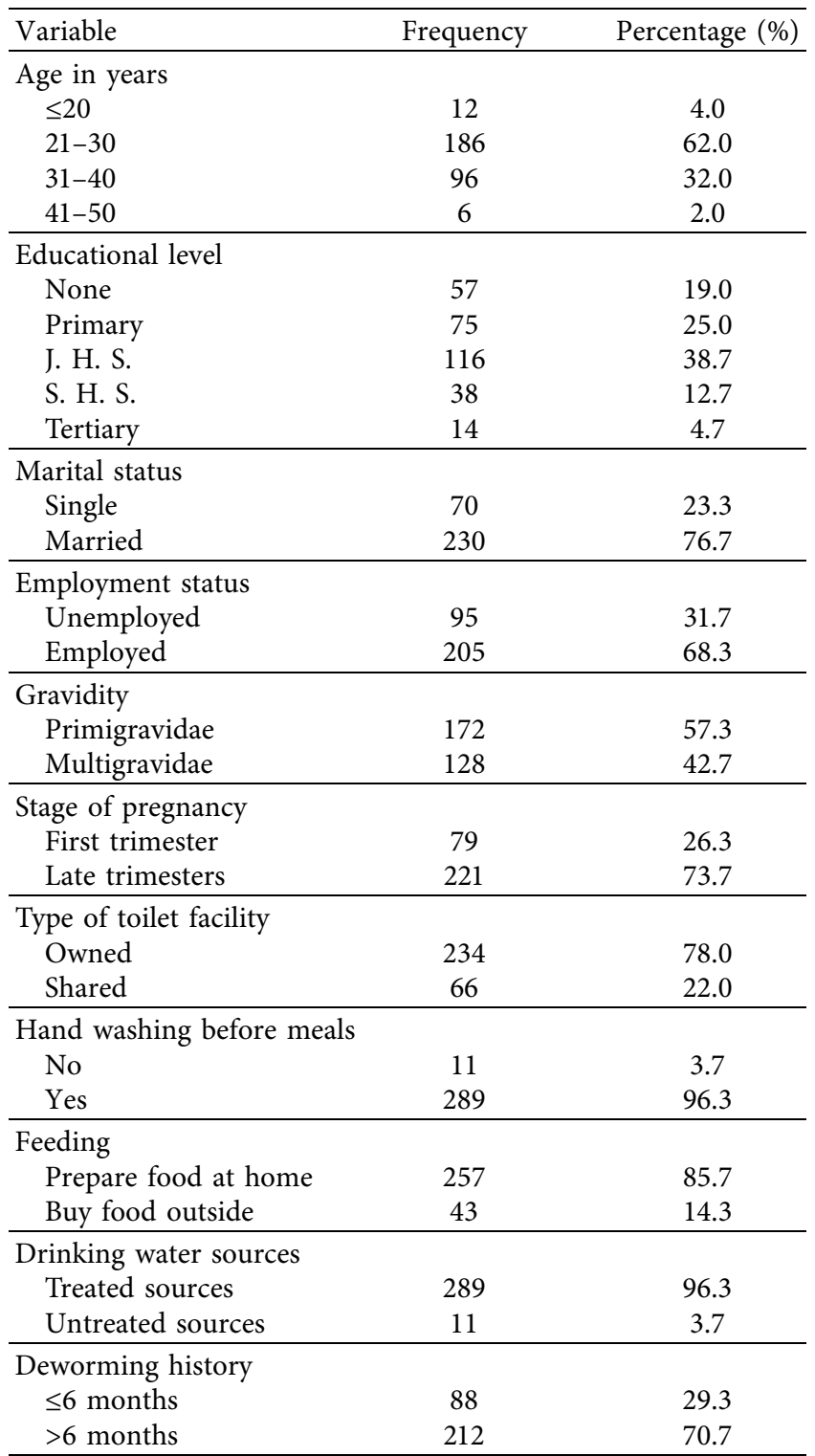

Data are presented as frequency and percentages; J. H. S.: junior high school; S. H. S.: senior high school.

$\mathrm{CI}=0.05-0.63 ; p=0.007)$. Also, pregnant women who drank water from untreated sources were 5.5 times likely of contracting intestinal parasitic infections $(\mathrm{COR}=5.50 ; 95 \%$ $\mathrm{CI}=1.60-18.92 ; p=0.007)$. Adjusting for potential confounding factors, gravidity was significantly associated with intestinal parasitic infections among pregnant women. There was a 57\% reduced odds of intestinal parasitic infections among multigravida women compared to their primigravidae counterparts $(\mathrm{AOR}=0.43 ; 95 \% \mathrm{CI}=0.19-0.97 ; p=0.043)$.

Meanwhile, the stage of pregnancy was a significant independent contributor of intestinal parasitic infections among pregnant women. The odds of intestinal parasitic infections among pregnant women in their late trimesters were 4.73 times higher compared to pregnant women in their first trimester $(\mathrm{AOR}=4.73 ; 95 \% \mathrm{CI}=1.36-16.49$; $p=0.015$ ) (Table 3).

\section{Discussion}

The overall prevalence of intestinal parasitic infections among pregnant women from the study was found to be $14.3 \%$ (95\% CI 11-19\%). This finding is similar to a prevalence of $14.32 \%$ obtained from a previous study in Northwest Ethiopia [17], $13.8 \%$ in Kenya [3], and $13.0 \%$ in Ghana [18]. Relatively higher prevalence rates have been reported in other studies, in Ghana (46.6\%) and Ethiopia $(70.6 \%)$, respectively $[5,8]$. In contrast, a study in Iran has reported a significantly lower infection rate of 3.73\% [19]. The differences in sociodemographic variables as well as hygienic practices are possible contributing factors to the prevalence of intestinal parasitic infections observed in the above literature.

Entamoeba histolytica contributed to the majority of intestinal parasites identified in the study representing a prevalence of $5.0 \%$. This finding differs from that of Forson et al. [20], which recorded a prevalence of $1 \%$ and ObiakorOkeke et al. [21] which recorded no parasites in their study. Though the findings contrast with that of the current study, Akinbo et al. [6] found E. histolytica and Plasmodium spp. coinfections prevalence to be $16.7 \%$ representing the most prevalent. Entamoeba histolytica was followed by Ascaris lumbricoides (4.3\%). The high prevalence of these two parasites (Entamoeba histolytica and Ascaris lumbricoides) poses a serious health threat to the pregnant women infected. These parasites are known to contribute to bleeding episodes and may lead to adverse pregnancy outcomes that can be life threatening.

With regard to Giardia lamblia, its prevalence was found to be $2.3 \%$ among the pregnant women. This finding is much higher compared to a prevalence of $1 \%$ reported by Akinbo et al. [6] but marginally lower than a prevalence of $2.6 \%$ observed by Obiakor-Okeke et al. [21] both in Nigeria.

Hookworm contributed to $0.3 \%$ of intestinal parasitic infections identified in the study. This results contrast with that of similar studies which reported higher prevalence of $3.0 \%, 7.0 \%$, and $38.6 \%$, respectively $[6,7,22]$. The low prevalence rate of hookworm infections observed in this study could be due to good sanitation and proper sewage disposal, as seen in the fact that many participants (78\%) had their own toilet facilities rather than shared.

Trichuris trichiura accounted for $1.3 \%$ of the infections. This was comparable to a prevalence of $1.3 \%$ obtained among pregnant women in Kenya [3] but slightly lower than a prevalence of $2.0 \%$ observed in Papua New Guinea [23].

Intestinal parasitic infection among pregnant women was determined by age, gravidity, and gestational age. Older age was associated with a reduction in intestinal parasitic infections. This may be due to older women having better knowledge on personal and environmental hygienic practices compared to pregnant women of lower ages. Other studies did not find any association between age and intestinal parasite infections among pregnant women $[7,24,25]$.

The stage of pregnancy/gestational age was also found to be associated with intestinal parasitic infections. The late trimesters (second and third trimesters) were associated 


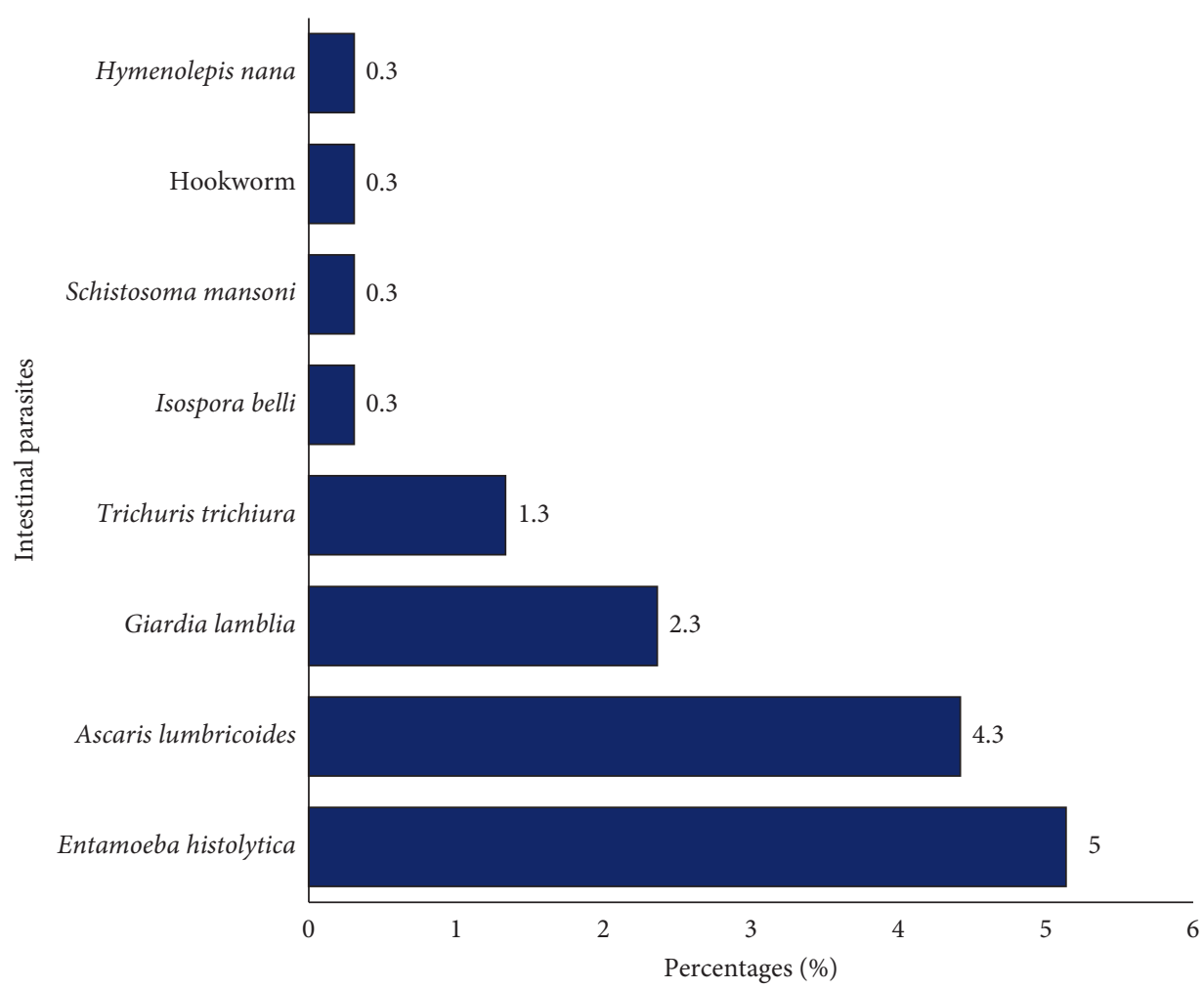

FIgURe 1: Prevalence of intestinal parasitic infections among pregnant women.

TABLE 2: Univariate and multivariate analysis of intestinal parasitic infections across sociodemographic characteristics of pregnant women in Kasoa Polyclinic.

\begin{tabular}{|c|c|c|c|c|c|c|}
\hline Characteristics & Number examined (\%) & Number of positive cases (\%) & COR (95\% CI) & $p$ value & AOR (95\% CI) & $p$ value \\
\hline \multicolumn{7}{|l|}{ Age } \\
\hline$\leq 30 \mathrm{yrs}$ & $198(66.0)$ & $38(19.2)$ & 1 & & 1 & \\
\hline$>30 \mathrm{yrs}$ & $102(34.0)$ & $5(4.9)$ & $0.22(0.83-1.57)$ & 0.002 & $0.17(0.06-0.48)$ & 0.001 \\
\hline \multicolumn{7}{|l|}{ Education } \\
\hline Less J. H. S. & $132(44.0)$ & $27(20.5)$ & 1 & & 1 & \\
\hline J. H. S. and above & $168(56.0)$ & $16(9.5)$ & $0.41(0.21-0.80)$ & 0.009 & $0.55(0.26-1.18)$ & 0.125 \\
\hline \multicolumn{7}{|l|}{ Marital status } \\
\hline Single & $70(23.3)$ & $9(12.9)$ & 1 & & - & \\
\hline Married & $230(76.7)$ & $34(14.8)$ & $1.18(0.53-2.59)$ & 0.688 & - & - \\
\hline \multicolumn{7}{|l|}{ Employment } \\
\hline Unemployed & $95(31.7)$ & $22(23.2)$ & 1 & & 1 & \\
\hline Employed & $205(68.3)$ & $21(10.2)$ & $0.38(0.20-0.73)$ & 0.004 & $0.49(0.23-1.05)$ & 0.066 \\
\hline
\end{tabular}

with increased odds of intestinal parasitic infections among women. This observation is consistent with previous findings where the second and third trimesters of pregnancy presented higher odds of intestinal parasite infections than their counterparts in their first trimester $[6,22]$. In contrast to these findings, Espinosa Aranzales and her colleagues have reported that stage of pregnancy had no association with intestinal parasitic infections [25].

Additionally, the study found gravidity to be associated with the odds of intestinal parasitic infections. Multigravida women had reduced odds of intestinal parasitism compared to primigravida women. The findings are in line with that of Phuanukoonnon et al. [23] and Yatich et al. [26]. This observation may be attributed to the fact that multigravida women have previous pregnancy experience and therefore may have benefitted from public health education on various practices to avoid intestinal parasitic infections.

However, none of the sanitation and hygienic practices was associated with intestinal parasitic infections among pregnant women. Whilst the findings are in line with those of Derso et al. [24] and Espinosa Aranzales et al. [25], they are contrary to the findings from Ethiopia, where hand washing practices, availability of toilet facilities, and feeding habits were found to be associated with intestinal parasite infections in pregnancy [5].

A major limitation to this study was the fact that a combination of Kato-Katz method (for helminth species) and formol-ether combination (for protozoa) would give a 
TABLE 3: Univariate and multivariate logistic regression analysis of intestinal parasitic infections with regard to gravidity, deworming status, and hygienic practices.

\begin{tabular}{|c|c|c|c|c|c|c|}
\hline Characteristics & Number examined (\%) & Number of positive cases (\%) & cOR $(95 \% \mathrm{CI})$ & $p$ value & aOR $(95 \% \mathrm{CI})$ & $p$ value \\
\hline \multicolumn{7}{|l|}{ Gravidity } \\
\hline Primigravidae & $172(57.3)$ & $31(18.0)$ & 1 & & 1 & \\
\hline Multigravidae & $128(42.7)$ & $12(9.4)$ & $0.47(0.23-0.96)$ & 0.037 & $0.43(0.19-0.97)$ & 0.043 \\
\hline \multicolumn{7}{|l|}{ Stage of pregnancy } \\
\hline First trimester & $79(26.3)$ & $3(3.8)$ & 1 & & 1 & \\
\hline Late trimesters & $221(73.7)$ & $40(18.1)$ & $5.60(1.68-18.65)$ & 0.005 & $4.73(1.36-16.49)$ & 0.015 \\
\hline \multicolumn{7}{|l|}{ Type of toilet facility } \\
\hline Owned & $234(78.0)$ & $26(11.1)$ & 1 & & 1 & \\
\hline Shared & $66(22.0)$ & $17(25.8)$ & $2.78(1.40-5.51)$ & 0.004 & $1.72(0.77-3.86)$ & 0.186 \\
\hline \multicolumn{7}{|c|}{ Hand washing before meals } \\
\hline No & $11(3.7)$ & $5(45.5)$ & 1 & & 1 & \\
\hline Yes & $289(96.3)$ & $38(13.2)$ & $0.18(0.05-0.63)$ & 0.007 & $0.25(0.06-1.03)$ & 0.055 \\
\hline \multicolumn{7}{|l|}{ Feeding } \\
\hline Prepare food at home & $257(85.7)$ & $34(13.2)$ & 1 & & - & \\
\hline Buy food outside & $43(14.3)$ & $9(20.9)$ & $1.74(0.77-3.94)$ & 0.187 & - & - \\
\hline \multicolumn{7}{|l|}{ Source of drinking water } \\
\hline Treated sources & $289(96.3)$ & $38(13.2)$ & 1 & & 1 & \\
\hline Untreated sources & $11(3.7)$ & $5(45.5)$ & $5.50(1.60-18.92)$ & 0.007 & $3.42(0.87-13.48)$ & 0.079 \\
\hline \multicolumn{7}{|l|}{ Deworming history } \\
\hline$\leq 6$ months & $88(29.3)$ & $8(9.1)$ & 1 & & - & \\
\hline$>6$ months & $212(70.7)$ & $35(16.5)$ & $1.977(0.88-4.46)$ & 0.100 & - & - \\
\hline
\end{tabular}

better estimate of prevalence. Again, PCR would have been more sensitive in the identification and the confirmation of several parasites but financial constraints limited our access to it.

\section{Conclusion}

In conclusion, the overall prevalence of intestinal parasitic infection was $14.3 \%$ among pregnant women. The major factors associated with this prevalence were age, gravidity, and gestational age. Therefore, there is need for continuous routine stool examination and health education to prevent infection of pregnant mothers and their unborn babies.

\section{Data Availability}

The data related to this submission are available on request.

\section{Conflicts of Interest}

The authors declare that they have no conflicts of interest.

\section{Acknowledgments}

The authors express their extreme gratitude to the staff and pregnant women of Kasoa Polyclinic who showed interest and participated in the research.

\section{References}

[1] J. Utzinger, S. L. Becker, S. Knopp et al., "Neglected tropical diseases: diagnosis, clinical management, treatment and control," Swiss Medical Weekly, vol. 142, 2012.
[2] S. Siziya, A. S. Muula, and E. Rudatsikira, "Correlates of diarrhoea among children below the age of 5 years in Sudan," African Health Sciences, vol. 13, no. 2, pp. 376-383, 2013.

[3] A. W. Wekesa, C. S. Mulambalah, C. I. Muleke, and R. Odhiambo, "Intestinal helminth infections in pregnant women attending antenatal clinic at Kitale district hospital, Kenya," Journal of Parasitology Research, vol. 2014, Article ID 823923, 5 pages, 2014.

[4] I. Yakasai and U. Umar, "A review of parasitic infestation in pregnancy," Asian Journal of Natural and Applied Sciences, vol. 2, no. 1, pp. 31-38, 2013.

[5] B. E. Feleke and T. H. Jember, "Prevalence of helminthic infections and determinant factors among pregnant women in Mecha district, northwest Ethiopia: a cross sectional study," BMC Infectious Diseases, vol. 18, no. 1, pp. 1-6, 2018.

[6] F. O. Akinbo, T. A. Olowookere, C. E. Okaka, and M. O. Oriakhi, "Co-infection of malaria and intestinal parasites among pregnant women in Edo State, Nigeria," Journal of Medicine in the Tropics, vol. 19, no. 1, pp. 43-48, 2017.

[7] G. Fuseini, D. Edoh, B. G. Kalifa, and D. Knight, "Plasmodium and intestinal helminths distribution among pregnant women in the Kassena-Nankana district of northern Ghana," International Journal of Nematology and Entomology, vol. 1, no. 3, pp. 37-41, 2013.

[8] S. C. K. Tay, E. A. Nani, and W. Walana, "Parasitic infections and maternal anaemia among expectant mothers in the Dangme east district of Ghana," BMC Research Notes, vol. 10, no. 1, pp. 1-9, 2017.

[9] S. Baidoo, S. C. Tay, and H. Abruquah, "Intestinal helminth infection and anaemia during pregnancy: a community based study in Ghana," African Journal of Microbiology Research, vol. 4, no. 16, pp. 17713-21718, 2010.

[10] D. G. Colley, A. L. Bustinduy, W. E. Secor, and C. H. King, "Human schistosomiasis," The Lancet, vol. 383, no. 9936, pp. 2253-2264, 2014. 
[11] W. Walana, S. Crowther, K. Tay, P. Tetteh, and J. B. Ziem, "Prevalence of intestinal protozoan infestation among primary school children in Urban and peri-urban communities in Kumasi," Ghana, vol. 2, no. 2, pp. 52-57, 2014.

[12] W. Walana, E. N. K. Aidoo, and S. C. K. Tay, "Prevalence of hookworm infection: a retrospective study in Kumasi," Asian Pacific Journal of Tropical Biomedicine, vol. 4, pp. 4S158-S161, 2014.

[13] P. Nyarko, "2010 population \& housing census," National Analytical Report, Ghana Statistical Service, Accra, Ghana, 2013.

[14] G. Porter, K. Hampshire, P. Kyei, M. Adjaloo, G. Rapoo, and K. Kilpatrick, "Linkages between livelihood opportunities and refugee-host relations: learning from the experiences of Liberian camp-based refugees in Ghana," Journal of Refugee Studies, vol. 21, no. 2, pp. 230-252, 2008.

[15] G. Fuseini, E. Edoh, B. Kalifa, A.-W. Hamid, and D. Knight, "Parasitic infections and anaemia during pregnancy in the Kassena-Nankana district of Northern Ghana," Journal of Public Health Epidemiology, vol. 2, no. 3, pp. 48-52, 2010.

[16] M. Cheesbrough, District Laboratory Practice in Tropical Countries, Part 1, Cambridge University Press, Cambridge, UK, 2006.

[17] M. Alem, B. Enawgaw, A. Gelaw, T. Kenaw, M. Seid, and Y. Olkeba, "Prevalence of anemia and associated risk factors among pregnant women attending antenatal care in azezo health center gondar town, northwest Ethiopia," Journal of Interdisciplinary Histopathology, vol. 1, no. 3, pp. 137-144, 2013.

[18] P. F. Ayeh-Kumi, S. A. Nti, K. O. Duedu et al., "Intestinal Parasitosis among pregnant women in Accra, Ghana," Ghana Journal of Allied Health Sciences, pp. 9-14, 2009.

[19] D. Balarak, M. J. Modrek, E. Bazrafshan, H. Ansari, and F. K. Mostafapour, "Prevalence of intestinal parasitic infection among food handlers in northwest Iran," Journal of Parasitology Research, vol. 2016, Article ID 8461965, 6 pages, 2016.

[20] A. O. Forson, I. Arthur, M. Olu-Taiwo, K. K. Glover, P. J. Pappoe-Ashong, and P. F. Ayeh-Kumi, "Intestinal parasitic infections and risk factors: a cross-sectional survey of some school children in a suburb in Accra, Ghana," $B M C$ Research Notes, vol. 10, no. 1, pp. 4-8, 2017.

[21] P. N. Obiakor-Okeke, T. C. Nwaogu, U. A. Agugo, and M. Odigwe Nkechi, "The prevalence of intestinal parasites and anaemia among pregnant women attending antenatal clinic in federal medical centre Owerri, Imo state," Nigeria, vol. 4, no. $25,2014$.

[22] J. C. Umeh, P. C. Inyang-Etoh, D. C. Okpokam, and I. B. OtuBassey, "Malaria and hookworm co-infection and their effects on anaemia of pregnant women attending ante-natal clinic in University Teaching Hospital, Calabar, Nigeria," Asian Journal of Medical Sciences, vol. 9, no. 3, pp. 27-35, 2018.

[23] S. Phuanukoonnon, A. Michael, W. Kirarock, W. S. Pomat, and A. H. J. Van den Biggelaar, "Intestinal parasitic infections and anaemia among pregnant women in the highlands of Papua New Guinea," Papua and New Guinea Medical Journal, vol. 56, no. 3-4, pp. 119-125, 2015.

[24] A. Derso, E. Nibret, and A. Munshea, "Prevalence of intestinal parasitic infections and associated risk factors among pregnant women attending antenatal care center at Felege Hiwot referral hospital, northwest Ethiopia," BMC Infectious Diseases, vol. 16, no. 1, pp. 1-7, 2016.

[25] A. F. Espinosa Aranzales, K. Radon, G. Froeschl, Á. María, P. Rondón, and M. Delius, "Prevalence and risk factors for intestinal parasitic infections in pregnant women residing in three districts of Bogotá, Colombia," BMC Public Health, vol. 18, no. 1, pp. 1-15, 2018.

[26] N. J. Yatich, J. C. Rayner, A. Turpin et al., "Malaria and intestinal helminth co-infection among pregnant women in Ghana: prevalence and risk factors," The American Journal of Tropical Medicine and Hygiene, vol. 80, no. 6, pp. 896-901, 2009. 